\title{
The Diagnosis and Treatment of the Early Changes in the Rheumatoid Wrist and Hand
}

\author{
L. SOLOMON, M.D., F.R.C.S. \\ Department of Orthopaedic Surgery and Rheumatism and \\ Arthritis Unit, Johannesburg Hospital
}

The wrist and hand are more commonly affected in rheumatoid arthritis than any other site in the body. Pulkki (1961) found that approximately one-third of patients with rheumatoid disease have tenosynovitis around the wrist and in the flexor sheaths of the fingers. Many more have synovitis of the wrist or metacarpophalangeal (M.C.P.) joints, often associated with periarticular erosions on the bone. Moreover, in 95 per cent of these patients involvement becomes bilateral with the inevitable progress of the disease (Short, Bauer and Reynolds, 1957).

Of 136 patients with definite rheumatoid arthritis seen in the Arthritis Clinic of the Johannesburg Hospital between January 1965 and December 1967, 103 had marked involvement of wrists and hands.

The classical deformities of the rheumatoid hand are too well known to warrant another detailed description (Fig. l). These are the late effects of the disease and treatment should be directed at preventing this grim progress towards painful deformity and loss of function. The anatomical distortions of the severely crippled hand have for so long held the limelight that a study of the early pathology of the disease has been neglected and treatment has come to mean salvage rather than prevention or cure.

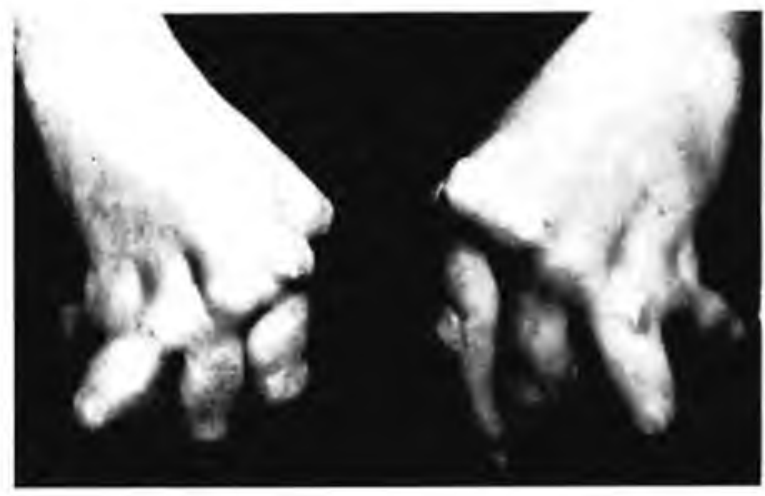

Fig. 1. The late deformities of the rheumatoid hand.

The present paper deals with the early lesions of the rheumatoid wrist and hand and their treatment by physical and surgical measures. Systemic treatment with salicylates and other anti-inflammatory agents may, of course, control the activity of the disease and check its progress in the hands as elsewhere. Indeed, the state of the interphalangeal joints is one of the more reliable measures of disease activity and is used to evaluate the efficacy of drug treatment (Boardman and Hart, 1967). In the majority of cases, however, these measures alone have proved inadequate and the hopes raised by each new "wonder drug" have always seemed brighter in newsprint than in clinical practice.

SYNOVITIS OF THE DISTAL RADIO-ULNAR JOINT

Synovitis of the distal radio-ulnar joint is frequently the earliest change in the wrist or hand. If not specifically sought for, however, it may be easily missed. There is generally a slight swelling around the joint and pressure on the ulnar head reveals a painful sub-luxation of the radioulnar joint. Radiographic examination may show the typical "cyst" or erosion of the ulnar styloid process or disruption of the joint itself (Fig. 2).

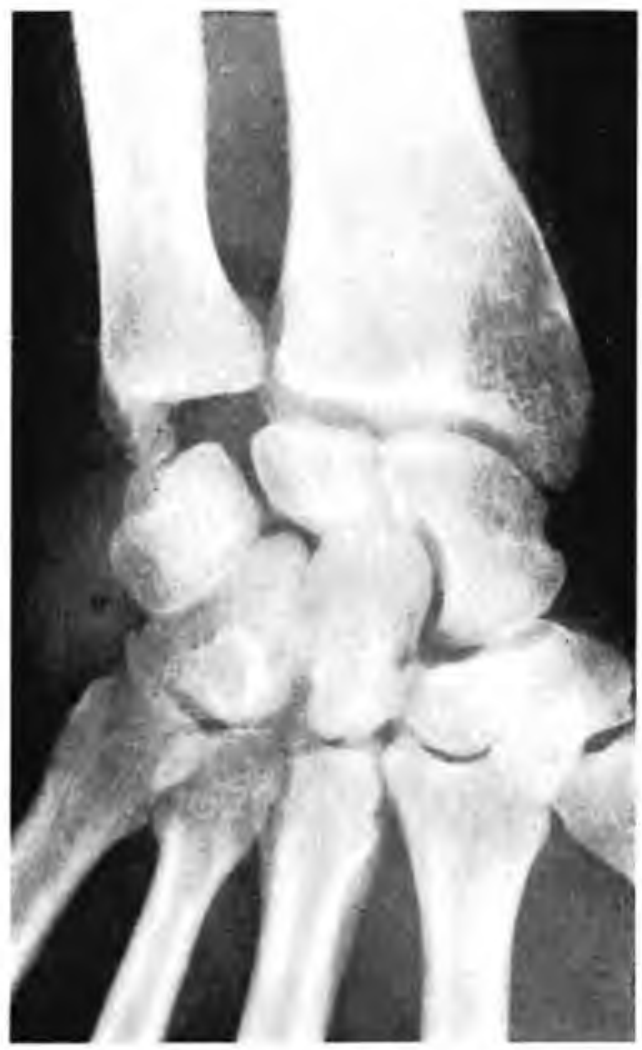

Fig. 2. Cystic erosion of the distal end of the ulna associated with synovitis of the radio-ulnar joint.

The diagnosis is important for this may be the first stage in a recognizable march of events: painful radio-ulnar joint-radial deviation of the carpus (Fig. 3) - ulnar deviation of the fingers to restore the alignment of the hand. It is significant that the vast majority of patients with established deformities of the fingers also have disease of the wrist with radial deviation of the carpus. Contrariwise, in Still's disease which is frequently associated with dysplasia of the distal end of the ulna and ulnar deviation of the carpus, there is usually no ulnar deviation of the fingers.

Treatment: Splinting of the wrist in the neutral position affords both rest for the inflamed joint and a gentle correction of the deformity. The resting splints, which are moulded out of plaster of Paris, duralumin or polyvinyl sheets, extend no further than the palm of the hand thus allowing full mobility of the metacarpophalangeal joints. They are usually 
worn only at night but, if the deformity progresses under treatment the splints may be worn day and night.

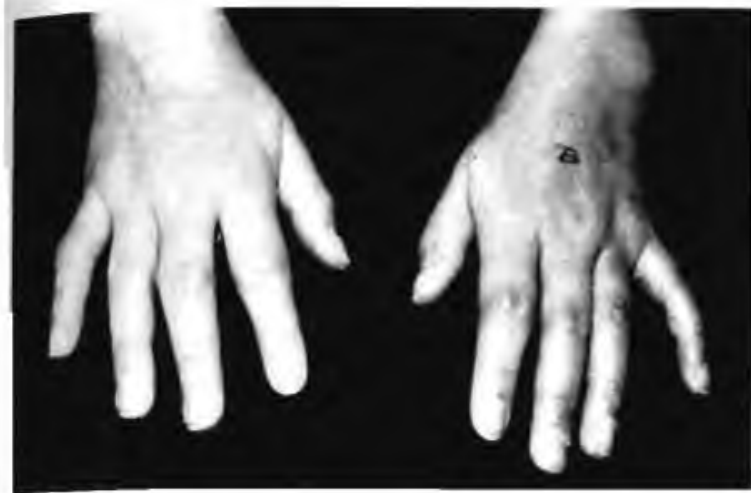

Fig. 3. Radial deviation of the wrist due to radio-ulnar synovitis.

If the radio-ulnar joint is still intact it is our practice to treat the synovitis by intra-articular injection of nitrogen mustard and betamethasone. Good results, with relief of pain and swelling for periods of $s^{i} x$ months to two years, have been obtained in at liast half of thess patients. For the later case, with dislocarion of the radio-ulner ju int, excision of the distal end of liev una and synovectomy of the urist is the treatment of choice.

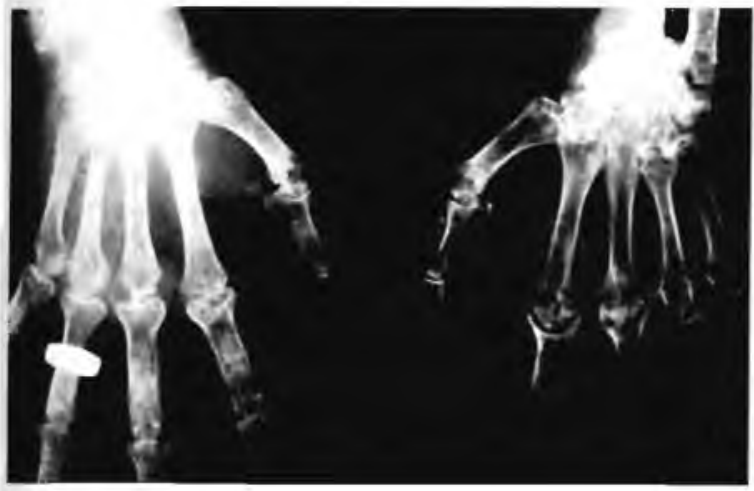

Fig. 4. The late effects of rheumatoid arthritis. Radiograph showing destruction of the carpus.

\section{SYNOVITIS OF THE RADIO-CARPAL JOINT}

Progressive involvement of the wrist may lead, ultimately, to destruction of the carpus (Fig. 4). In the early stages, however, pain due to acute and sub-acute synovitis may be out of all proportion to the detectable damage to the joint. Stability of the wrist is all-important in maintaining control and power in the hand and not infrequently the weak grip of the rheumatoid hand is exaggerated by pain in the wrist.

Treatment: Where good mobility of the wrist is retained and the radiographs show an intact articular surface, treatment is the same as that for radio-ulnar synovitis. Relief of symptoms and signs for a year or longer has been achieved in the majority of these patients but ultimately two out of every three have required surgical synovectomy. As a rule this is combined with synovectomy of the extensor tendons and excision of the distal end of the ulna. Post-operatively the wrist is splinted firmly for two weeks and then gradual movement is commenced. By the end of six weeks full movement is generally regained.

\section{SYNOVITIS OF THE METACARPO-PHALANGEAL (M.C.P.) JOINTS}

The swollen M.C.P. joints are the classic trade-mark of the rheumatoid hand, recognised by specialist and layman alike as the sign of a "spreading arthritis" (Fig. 5). For the patient the early morning swelling and stiffness of the hands may be the major-or indeed the only-disability for several years. For the rheumatologist synovitis of the M.C.P. and proximal interphalangeal (P.I.P.) joints, with periarticular erosions and osteoporosis, may be the earliest sign of rheumatoid disease, often preceding the discovery of positive serological reactions. It is at this stage that treatment can be most effective in preventing the late results of M.C.P. arthritis. The combination of chronic synovitis, swelling of the joint and articular erosion by granulation tissue will, if left unchecked, lead inexorably to cartilage degeneration, stretching of the capsule and ligaments and finally disruption of the joint.

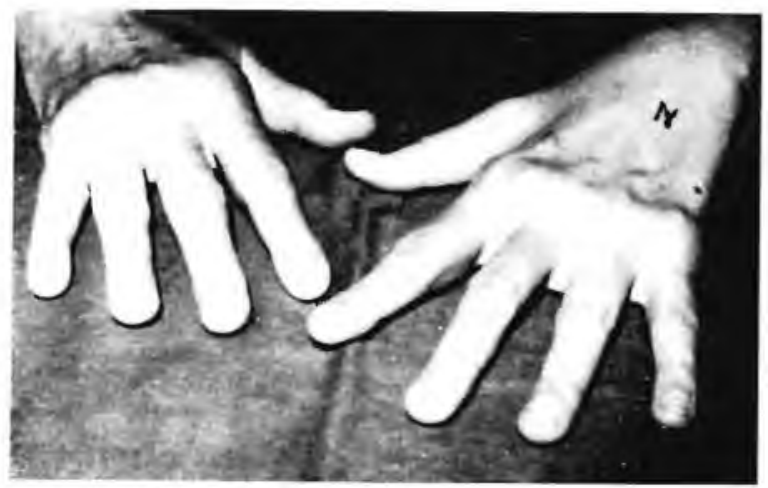

Fig. 5. Synovitis and swelling of the M.C.P. joints.

In the overwhelming majority of such cases it is the second and third M.C.P. joints that are affected first. Together with the radio-carpal joint they control the radial pillar of the hand; instability of these articulations permits the mechanical distortions due to muscular weakness, tendon rupture or the repetitive forces of grip and pressure that accompany the most ordinary activities.

Treatment: The best hope of preventing the late crippling deformities of the rheumatoid hand lies in the early and effective treatment of the M.C.P. synovitis. Systemic treatment during the active phase of the disease may bring about a reduction in the swelling of the joints. As a rule, however, we are loath to wait for this uncertain outcome; if there is an obvious synovitis with visible and palpable joint swelling, systemic treatment is combined with a direct attack upon the joints.

The results of intra-articular injection of nitrogen mustard and betamethasone have encouraged us to use this as a first measure in every new case. The mixture used contains $20 \mathrm{mg}$. betamethasone per $\mathrm{ml}$. and nitrogen mustard in a concentration of 0.002 per cent. Just enough is injected to distend the joint-usually 0.5 to $1 \mathrm{ml}$. - and if necessary, all the M.C.P. joints are injected at the same sitting.

Twenty-four hours after injection ice treatment of the hands is commenced and movements are encouraged. At night, however, the hands are rested in moulded splints with the M.C.P. joints in the neutral position. This is continued until pain and swelling subside.

The result of this treatment in 22 new cases ( 68 joints) have been satisfactory in 75 per cent, the remission of pain and swelling lasting for periods of six months to two years. Failure to reduce the synovitis is regarded as an indication for surgical synovectomy of the M.C.P. joints. If the ulnar drift of the fingers has already commenced, synovectomy 
is combined with plication of the joint capsule and realignment of the fingers in the neutral position.

\section{EXTENSOR TENOSYNOVITIS AT THE WRIST}

Tenosynovitis of the extensor tendons usually accompanies synovitis of the wrist joint. It may, however, prove to be the more important aspect of any particular case, for a persistent tenosynvitis with inflammatory erosion of the extensor tendons is a frequent cause of tendon rupture (Fig. 6).

Treatment: Neither local injections nor splints are of much avail in these cases and synovectomy is usually called for. This may be combined with synovectomy of the wrist and excision of the distal end of the ulna.

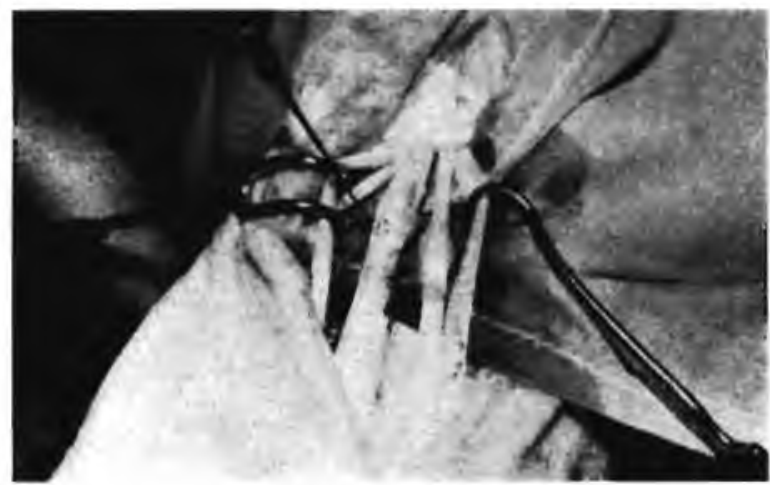

Fig. 6. Extensor tenosynovitis at the wrist. Nodular degeneration of the tendon is shown.

FLEXOR TENOSYNOVITIS AT THE WRIST

The effects of flexor tenosynovitis are similar to those of extensor tenosynovitis. In addition, however, the bulky synovial tissue may cause median nerve compression in the carpal tunnel. Paraesthesiae and numbness in the median nerve distribution, or weakness of the short abductor of the thumb, should immediately suggest this diagnosis.

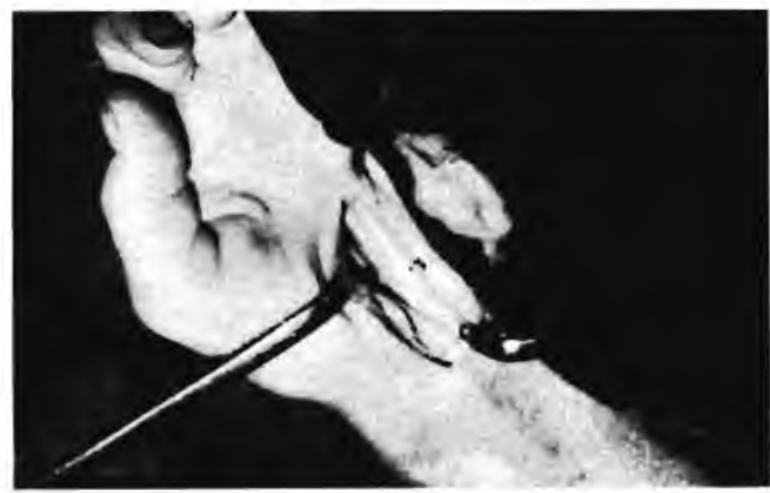

Fig. 7. Synovectomy and decompression of the carpal tunnel.

Treatment: Median nerve decompression is achieved by division of the transverse carpal ligament and a careful synovectomy of the flexor tendons as they cross the wrist (Fig. 7). After operation the hand and wrist are splinted for 48 hours and thereafter movements are encouraged.

\section{FLEXOR TENOSYNOVITIS OF THE FINGERS}

It is usually taken for granted that pain and stiffness of the fingers in rheumatoid disease are due to a polyarthritis. This betrays a failure to examine the hand carefully for a tenosynovitis of the flexor tendons. The records of the
Arthritis Clinic show that the latter is a major factor in no less than 30 per cent of patients with pain and stiffness of the hands

A thumb placed over the flexor tendon in the palm wil discover the typical crepitation on passive flexion and extension of the affected finger. A similar, but less obvious, disturbance may be found at the level of the P.I.P. joint where the flexor digitorum profundus passes through the slips of the superficial flexor. In the worst cases the thickened sheath may be palpated along the length of the proximal phalanx.

Interference with the gliding mechanism of the flexor tendon is demonstrated by the patient's inability to curl the affected finger into the palm of the hand (Fig. 8). This further weakens the grip and contributes significantly to the vulnerability of the M.C.P. joints to mechanical distortion.

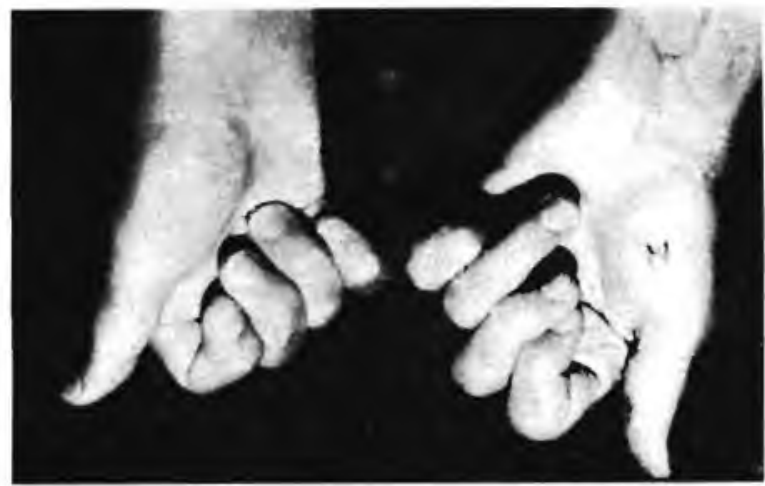

Fig. 8. Flexor tenosynovitis of the hand and fingers causing inability to curl the affected fingers into full flexion.

Treatment: If the diagnosis is made sufficiently early local injection of nitrogen mustard and betamethasone into the flexor sheaths usually produces a complete remission of the synovit is. In the long standing case, however-and especially in those with nodules on the flexor tendons - surgical synovectomy is the treatment of choice. No splints are applied after this procedure and early movements are encouraged in order to re-establish the free gliding action of the flexor tendons in the palm and fingers.

Flexor synovectomy has been carried out in 18 patients. Twelve of these (17 hands) have been followed for at least one year after operation. In all but two of these patients there has been an improvement in the range of finger movement and in eight the operation has restored nearnormal mobility (Fig. 9).

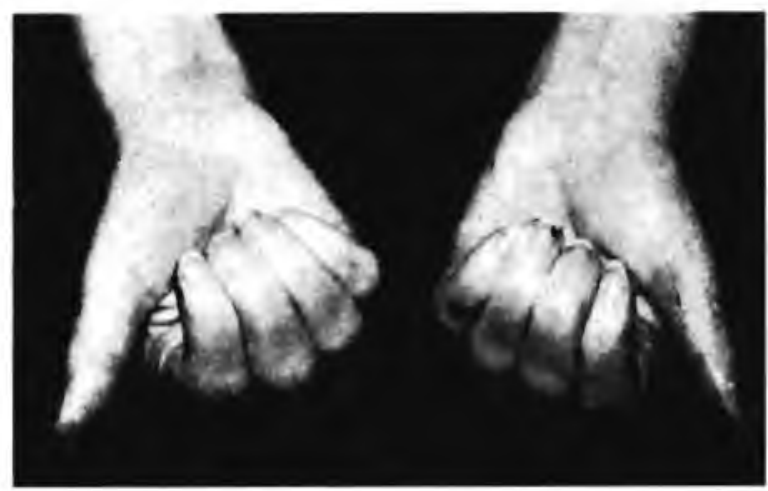

Fig. 9. Finger flexion after flexor synovectomy.

(continued on page 5.) 